PROCEEDINGS OF THE

AMERICAN MATHEMATICAL SOCIETY

Volume 127, Number 7, Pages 2083-2090

S 0002-9939(99)05150-3

Article electronically published on March 16, 1999

\title{
EXTENSIONS OF A THEOREM OF MARCINKIEWICZ-ZYGMUND AND OF ROGOSINSKI'S FORMULA AND AN APPLICATION TO UNIVERSAL TAYLOR SERIES
}

\author{
E. S. KATSOPRINAKIS AND M. PAPADIMITRAKIS
}

(Communicated by Albert Baernstein II)

\begin{abstract}
This paper extends Rogosinski's formula and the MarcinkiewiczZygmund Theorem about circular structure of the limit points of the partial sums of $(\mathrm{C}, 1)$ summable Taylor series. Also a result about summability of $H^{p}$ Taylor series is proved and an application on Universal Taylor series is given.
\end{abstract}

\section{INTRODUCTION}

Let $\sum_{n=0}^{\infty} a_{n} z^{n}, a_{n} \in \mathbb{C}$, be a power series, convergent for $|z|<1$. A classical theorem of Marcinkiewicz-Zygmund (see [2], [5], [9], Vol. II, p. 178) says that, if this series is $(\mathrm{C}, 1)$ summable at every point $z$ of a subset $E$ of the unit circle $T=\{z \in \mathbb{C}:|z|=1\}$, then, for almost every $z$ in $E$, the set of limit points of the partial sums of the series has circular structure with center the $(\mathrm{C}, 1)$ sum.

One of the results of this paper is an extension of the just mentioned theorem to $(\mathrm{C}, k)$ summability with $k \geq 1$. This is Theorem 1 in section 2 . This result came as an immediate consequence of an extention of the main ingredient in the proof of the theorem of Marcinkiewicz-Zygmund, namely the formula of Rogosinski (see [2], [9], Theorem 12.16, Ch. III). We extend this formula in Theorem 2 of section 2.

Our work on the formula of Rogosinski was motivated by our desire to answer certain questions on the subject of Universal Taylor series (see [7]); more precisely, whether such a series can be $(\mathrm{C}, k)$ summable on its circle of convergence and whether it can belong to any of the Hardy spaces $H^{p}$. It was J. -P. Kahane who suggested the above extension of Rogosinski's formula in order to answer these questions. The results related to this subject are contained in section 3.

Section 4 contains remarks and some further comments.

\section{MAin RESUlts}

Let $S(z)=\sum_{n=0}^{\infty} a_{n} z^{n}$ be a power series. We denote by $S_{N}(z)=\sum_{n=0}^{N} a_{n} z^{n}$ the partial sums of this series, and, generally, by $S_{N}^{(k)}(z)$ its $(\mathrm{C}, k)$ sums. These are

Received by the editors October 13, 1997.

1991 Mathematics Subject Classification. Primary 30B30; Secondary 41A58, 42A24, 30E10.

(C)1999 American Mathematical Society 
defined inductively (for integer $k$ ) by

$$
\begin{gathered}
S_{N}^{(0)}(z)=S_{N}(z), \\
S_{N}^{(k+1)}(z)=S_{0}^{(k)}(z)+\cdots+S_{N}^{(k)}(z) .
\end{gathered}
$$

In the particular case of the constant power series 1 (which means $a_{0}=1, a_{1}=$ $a_{2}=\cdots=0$ ) the corresponding sums are denoted by $A_{N}^{(k)}$. Hence

$$
\begin{gathered}
A_{N}^{(0)}=1, \\
A_{N}^{(k+1)}=A_{0}^{(k)}+\cdots+A_{N}^{(k)} .
\end{gathered}
$$

It is easy to see that $A_{N}^{(k)}=\left(\begin{array}{c}N+k \\ N\end{array}\right) \sim \frac{N^{k}}{\Gamma(k+1)}$, as $N \rightarrow \infty$.

By $\sigma_{N}^{(k)}(z)$ we denote the $(\mathrm{C}, k)$ means of the series, defined by

$$
\sigma_{N}^{(k)}(z)=\frac{S_{N}^{(k)}(z)}{A_{N}^{(k)}} .
$$

We say that $S(z)$ is $(\mathrm{C}, k)$ summable at the point $z$ and that it has $\sigma^{(k)}(z)$ as its $(\mathrm{C}, k)$ sum, if $\sigma_{N}^{(k)}(z) \rightarrow \sigma^{(k)}(z)$, as $N \rightarrow \infty$.

All this is classical and the basic terminology and facts concerning $(\mathrm{C}, k)$ summability are described in [1] and [9]. For simplicity we restrict ourselves to the case of integral $k$. Our first main result is the following:

Theorem 1. Let the power series $S(z)$ converge for $|z|<1$. Also let it be $(C, k)$ summable for every $z$ in a certain subset $E$ of the unit circle $T$, with $(C, k)$ sum $\sigma^{(k)}(z)$. Then, for almost every $z$ of $E$, the set $L(z)$ of limit points of the sequence

$$
\frac{S_{N}(z)-\sigma^{(k)}(z)}{N^{k-1}}, \quad N=1,2,3, \ldots,
$$

has circular structure with center 0 .

A set $L$ in $\mathbb{C}$ has circular structure with center $\alpha$ if, for every $z$ in $L$, the whole circle $\{\zeta:|\zeta-\alpha|=|z-\alpha|\}$ belongs to $L$.

The theorem of Marcinkiewicz-Zygmund is the special case $k=1$ of Theorem 1 . Observe that, for $k \geq 2$, the actual value of $\sigma^{(k)}(z)$ plays no role in the structure of $L(z)$.

The proof of Theorem 1, as we mentioned in the Introduction, depends heavily on the following extension of the formula of Rogosinski:

Theorem 2. Let $S(z)$ be convergent for $|z|<1$ and be $(C, k)$ summable at $z_{0}$, with $\left|z_{0}\right|=1$. Let $\left\{z_{N}\right\}$ be a sequence with $z_{N}-z_{0}=O\left(\frac{1}{N}\right)$. Then,

$$
\begin{gathered}
S_{N}\left(z_{N}\right)-\sigma^{(k)}\left(z_{0}\right) \\
=\left(\frac{z_{N}}{z_{0}}\right)^{N} \sum_{\mu=0}^{k}\left(1-\frac{z_{0}}{z_{N}}\right)^{\mu} \sum_{m=\mu}^{k}\left(\begin{array}{c}
k-\mu \\
m-\mu
\end{array}\right)(-1)^{m} A_{N-m}^{(k)}\left(\sigma_{N-m}^{(k)}\left(z_{0}\right)-\sigma^{(k)}\left(z_{0}\right)\right)+o(1),
\end{gathered}
$$

as $N \rightarrow \infty$.

One trivially sees that, when $k=1$, the formula of Theorem 2 becomes

$$
S_{N}(z)-\sigma^{(1)}(z)=\left(\frac{z_{N}}{z_{0}}\right)^{N}\left(S_{N}\left(z_{0}\right)-\sigma^{(1)}\left(z_{0}\right)\right)+o(1),
$$

which is identical to Theorem 12.16, Ch. III in [9] (Rogosinski's formula) with a slight difference. In Rogosinski's formula $z_{0}=e^{i x}, z_{N}=e^{i\left(x+a_{N}\right)}$ with $a_{N}=O\left(\frac{1}{N}\right)$, 
i.e. $z_{N}$ is confined on the unit circle $T$. Here we allow $z_{N}$ to go out of $T$. The possibility of doing this was initially observed by V. Nestoridis.

Proof of Theorem 2. Without loss of generality we assume that $z_{0}=1$ and we supress it from all occurrences, i.e. $\sigma^{(k)}=\sigma^{(k)}(1), S_{N}^{(k)}=S_{N}^{(k)}(1)$ etc. Hence $a_{n}=S_{n}^{(0)}-S_{n-1}^{(0)}\left(\right.$ where of course $\left.S_{-1}^{(0)}=0\right)$, and with repeated summations by parts we find:

$$
\begin{aligned}
& S_{N}\left(z_{N}\right)=\sum_{n=0}^{N} a_{n} z_{N}^{n}=S_{N}^{(0)} z_{N}^{N}+\left(1-z_{N}\right) \sum_{n=0}^{N-1} S_{n}^{(0)} z_{N}^{n} \\
= & S_{N}^{(0)} z_{N}^{N}+S_{N-1}^{(1)} z_{N}^{N-1}\left(1-z_{N}\right)+\left(1-z_{N}\right)^{2} \sum_{n=0}^{N-2} S_{n}^{(1)} z_{N}^{n}
\end{aligned}
$$

and finally:

$$
\begin{aligned}
S_{N}\left(z_{N}\right) & =S_{N}^{(0)} z_{N}^{N}+S_{N-1}^{(1)} z_{N}^{N-1}\left(1-z_{N}\right)+\cdots+S_{N-k}^{(k)} z_{N}^{N-k}\left(1-z_{N}\right)^{k} \\
& +\left(1-z_{N}\right)^{k+1} \sum_{n=0}^{N-k-1} S_{n}^{(k)} z_{N}^{n} .
\end{aligned}
$$

The same formula applied to the constant series 1 implies:

$$
\begin{aligned}
1 & =A_{N}^{(0)} z_{N}^{N}+A_{N-1}^{(1)} z_{N}^{N-1}\left(1-z_{N}\right)+\cdots+A_{N-k}^{(k)} z_{N}^{N-k}\left(1-z_{N}\right)^{k} \\
& +\left(1-z_{N}\right)^{k+1} \sum_{n=0}^{N-k-1} A_{n}^{(k)} z_{N}^{n} .
\end{aligned}
$$

Multiplying (2) by $\sigma^{(k)}$ and substracting from (1) we get:

$$
\begin{aligned}
S_{N}\left(z_{N}\right)-\sigma^{(k)} & =\sum_{\mu=0}^{k}\left(S_{N-\mu}^{(\mu)}-\sigma^{(k)} A_{N-\mu}^{(\mu)}\right) z_{N}^{N-\mu}\left(1-z_{N}\right)^{\mu} \\
& +\left(1-z_{N}\right)^{k+1} \sum_{n=0}^{N-k-1}\left(S_{n}^{(k)}-\sigma^{(k)} A_{n}^{(k)}\right) z_{N}^{n} .
\end{aligned}
$$

Now consider the last sum in (3) i.e.

$$
\left(1-z_{N}\right)^{k+1} \sum_{n=0}^{N-k-1}\left(S_{n}^{(k)}-\sigma^{(k)} A_{n}^{(k)}\right) z_{N}^{n}=\left(1-z_{N}\right)^{k+1} \sum_{n=0}^{N-k-1} A_{n}^{(k)} z_{N}^{n}\left(\sigma_{n}^{(k)}-\sigma^{(k)}\right) .
$$

It can be considered as a "Toeplitz mean" of the sequence $\left\{\sigma_{n}^{(k)}-\sigma^{(k)}\right\}$. This sequence tends to 0 and the two properties of the "coefficients":

- $\left(1-z_{N}\right)^{k+1} A_{n}^{(k)} z_{N}^{n} \rightarrow 0$, as $N \rightarrow \infty$, for fixed $n$

- $\left|1-z_{N}\right|^{k+1} \sum_{n=0}^{N-k-1}\left|A_{n}^{(k)}\right|\left|z_{N}^{n}\right| \leq\left(\frac{M}{N}\right)^{k+1} \sum_{n=0}^{N-k-1} c n^{k}\left(1+\frac{M}{N}\right)^{n} \leq c M^{k+1} e^{M}$ (c, $M$ are absolute constants), guarantee that the last sum of (3) is $o(1)$, as $N \rightarrow \infty$. 
Next, if $\mu<k$, we get:

$$
\begin{aligned}
S_{n}^{(\mu)} & =S_{n}^{(\mu+1)}-S_{n-1}^{(\mu+1)}=S_{n}^{(\mu+2)}-2 S_{n-1}^{(\mu+2)}+S_{n-2}^{(\mu+2)} \\
& =\cdots=\sum_{m=0}^{k-\mu}\left(\begin{array}{c}
k-\mu \\
m
\end{array}\right)(-1)^{m} S_{n-m}^{(k)}
\end{aligned}
$$

and the same formula for $A_{n}^{(\mu)}$.

Replacing (4) and the similar formula for $A_{n}^{(\mu)}$ in the first sum of (3) and taking into account that the last sum of (3) is $o(1)$ we get:

$$
\begin{gathered}
S_{N}\left(z_{N}\right)-\sigma^{(k)} \\
=\sum_{\mu=0}^{k} \sum_{m=0}^{k-\mu}\left(\begin{array}{c}
k-\mu \\
m
\end{array}\right)(-1)^{m}\left(S_{N-\mu-m}^{(k)}-\sigma^{(k)} A_{N-\mu-m}^{(k)}\right) z_{N}^{N-\mu}\left(1-z_{N}\right)^{\mu}+o(1) \\
=z_{N}^{N} \sum_{\mu=0}^{k}\left(1-\frac{1}{z_{N}}\right)^{\mu} \sum_{m=\mu}^{k}\left(\begin{array}{c}
k-\mu \\
m-\mu
\end{array}\right)(-1)^{m} A_{N-m}^{(k)}\left(\sigma_{N-m}^{(k)}-\sigma^{(k)}\right)+o(1)
\end{gathered}
$$

and this proves Theorem 2 .

Proof of Theorem 1. Using $z_{N}=z_{0}, \quad N=0,1,2, \ldots$, in the formula of Theorem 2 one finds:

$$
S_{N}\left(z_{0}\right)-\sigma^{(k)}\left(z_{0}\right)=\sum_{m=0}^{k}\left(\begin{array}{c}
k \\
m
\end{array}\right)(-1)^{m} A_{N-m}^{(k)}\left(\sigma_{N-m}^{(k)}\left(z_{0}\right)-\sigma^{(k)}\left(z_{0}\right)\right)+o(1) .
$$

This is the term $\mu=0$ of the sum in the same formula. Therefore

$$
\begin{gathered}
\frac{S_{N}\left(z_{N}\right)-\sigma^{(k)}\left(z_{0}\right)}{N^{k-1}}=\left(\frac{z_{N}}{z_{0}}\right)^{N} \frac{S_{N}\left(z_{0}\right)-\sigma^{(k)}\left(z_{0}\right)}{N^{k-1}} \\
+\left(\frac{z_{N}}{z_{0}}\right)^{N} \sum_{\mu=1}^{k}\left(1-\frac{z_{0}}{z_{N}}\right)^{\mu} \frac{1}{N^{k-1}} \sum_{m=\mu}^{k}\left(\begin{array}{c}
k-\mu \\
m-\mu
\end{array}\right)(-1)^{m} A_{N-m}^{(k)}\left(\sigma_{N-m}^{(k)}\left(z_{0}\right)-\sigma^{(k)}\left(z_{0}\right)\right) \\
+o\left(\frac{1}{N^{k-1}}\right) .
\end{gathered}
$$

The last sum is, in absolute value, less than or equal to

$$
c\left(1+\frac{M}{N}\right)^{N} \sum_{\mu=1}^{k}\left(\frac{M}{N}\right)^{\mu} \frac{1}{N^{k-1}} \sum_{m=\mu}^{k}(N-m)^{k}\left|\sigma_{N-m}^{(k)}\left(z_{0}\right)-\sigma^{(k)}\left(z_{0}\right)\right|=\sum_{\mu=1}^{k} o\left(\frac{1}{N^{\mu-1}}\right),
$$

which is $o(1)$, as $N \rightarrow \infty$. Therefore,

$$
\frac{S_{N}\left(z_{N}\right)-\sigma^{(k)}\left(z_{0}\right)}{N^{k-1}}=\left(\frac{z_{N}}{z_{0}}\right)^{N} \frac{S_{N}\left(z_{0}\right)-\sigma^{(k)}\left(z_{0}\right)}{N^{k-1}}+o(1), \text { as } N \rightarrow \infty .
$$

Next, let $z_{0}=e^{i x}, z_{N}=e^{i\left(x+\beta_{N}\right)}, \beta_{N}=O\left(\frac{1}{N}\right)$. Then,

$$
\frac{S_{N}\left(x+\beta_{N}\right)-\sigma^{(k)}(x)}{N^{k-1}}=e^{i N \beta_{N}} \frac{S_{N}(x)-\sigma^{(k)}(x)}{N^{k-1}}+o(1) .
$$

Assuming $k \geq 2$ (for $k=1$ we have the Marcinkiewicz-Zygmund Theorem) and setting

$$
t_{N}(x)=\frac{1}{N^{k-1}} S_{N}(x)
$$


we find:

$$
t_{N}\left(x+\beta_{N}\right)=e^{i N \beta_{N}} t_{N}(x)+o(1), \text { as } N \rightarrow \infty .
$$

Now the rest of the proof is identical to the proof of the Marcinkiewicz- Zygmund Theorem (see [9], Vol. II, p. 178). Only for the sake of completeness (and because it is not so well known) we give here a sketch of proof.

Denote by $D(\zeta, r)$ the open disk centered at $\zeta$ with radius $r$, and by $A\left(r_{1}, r_{2}\right)$ the open ring centered at 0 with extremal radii $r_{1}$ and $r_{2}$. Remember that, for every $x \in E, S(x)$ is $(\mathrm{C}, k)$ summable and this implies (5) whenever $\beta_{n}=O\left(\frac{1}{n}\right)$. To prove that $L(x)$ has circular structure for almost every $x$ in $E$, it is enough to prove that, if $D(\zeta, r)$ is any disc with rational center $\zeta \neq 0$ and rational radius $r \leq|\zeta|$, then for almost every $x \in E$ : if $L(x)$ does not cut $D(\zeta, r)$, then it does not cut $A(|\zeta|-r,|\zeta|+r)$ either. Now consider some increasing sequence of radii tending to $r, r_{k} \uparrow r$. Consider also the set $E_{k, N}$ of all $x \in E$ such that: $t_{n}(x)$ is not in $D\left(\zeta, r_{k}\right)$ for every $n \geq N$. It is enough to prove that for almost every $x \in E_{k, N}$ the set $L(x)$ does not cut $A\left(|\zeta|-r_{k},|\zeta|+r_{k}\right)$.

Take any point of density $x$ of $E_{k, N}$. If $L(x)$ cuts $A\left(|\zeta|-r_{k},|\zeta|+r_{k}\right)$ then, for some sequence of $n$ 's, $t_{n}(x)$ will tend to some point of $A\left(|\zeta|-r_{k},|\zeta|+r_{k}\right)$ making an angle, say $\gamma$, with $\zeta$. Find a sequence $\beta_{n}$ such that:

$$
\text { (i) } x+\beta_{n} \in E_{k, N} \text { and (ii) } n \beta_{n} \rightarrow-\gamma \text {. }
$$

Then (i) implies that $t_{n}\left(x+\beta_{n}\right)$ is not in $D\left(\zeta, r_{k}\right)$ for all $n \geq N$, while (ii), together with (5), implies that $t_{n}\left(x+\beta_{n}\right)$ is in $D\left(\zeta, r_{k}\right)$ for a sequence of $n$ 's.

Thus we arrive at a contradiction and we finish the proof of Theorem 1.

Note that a result of M. Riesz (see [1], Theorem 76) immediately implies that, if $\sum_{n=0}^{\infty} a_{n} z^{n}$ is $(\mathrm{C}, k)$ summable, then $\sum_{n=0}^{\infty} \frac{a_{n}}{(n+1)^{k-1}} z^{n}$ is $(\mathrm{C}, 1)$ summable. Therefore, by the Marcinkiewicz-Zygmund Theorem the limit points of the partial sums $\sum_{n=0}^{N} \frac{a_{n}}{(n+1)^{k-1}} z^{n}$ have, for almost every $z \in E$, circular structure around the $(\mathrm{C}, 1)$ sum of the last series (which depends on $z$ ).

\section{An application}

A Taylor series $\sum_{n=0}^{\infty} a_{n} z^{n}$ with radius of convergence equal to 1 is called Universal if, on any compact subset $K$ of the complex plane not intersecting the open unit disc and with connected complement, its partial sums approximate uniformly any given function continuous on $K$ and holomorphic in the interior of $K$.

This definition is due to $\mathrm{V}$. Nestoridis who proved the existence and the basic properties of such series in the framework of a project studying the behaviour of partial sums of Taylor series (see [7], [8]).

Natural questions arise about this class of series. Here we answer two of them by the following:

Theorem 3. A Universal Taylor series cannot be $(C, k)$ summable at any point of its circle of convergence. Also it cannot belong to any $H^{p}$ space, $p>0$.

Note that [6] contains the result that any Universal Taylor series cannot belong to the class $N$ of Nevanlinna, thus implying the last part of our Theorem 3 . But 
since the method of proof is different and since it may have some independent interest we include it here.

Proof of Theorem 3. Let $S(z)=\sum_{n=0}^{\infty} a_{n} z^{n}$ be a Universal Taylor series. Assume that it is $(\mathrm{C}, k)$ summable at a certain point $z_{0},\left|z_{0}\right|=1$. Let $K=\{\zeta:|\zeta| \geq$ $\left.1,\left|\zeta-z_{0}\right| \leq \delta\right\}$ for small $\delta>0$. Consider the constant function $\sigma^{(k)}\left(z_{0}\right)+1$ on $K$ and (since $S(z)$ is Universal) a subsequence of $S_{N}$ 's converging uniformly on $K$ towards this constant. Let

$$
z_{N}=\frac{z_{0}}{1-\frac{x}{N}}, \text { where } x>0, N>x .
$$

Then, the formula of Theorem 2 implies, for this subsequence of $N$ 's, that

$$
e^{-x}=\lim _{N} \sum_{\mu=0}^{k} x^{\mu} A_{\mu, N}, \quad x>0,
$$

where

$$
A_{\mu, N}=\frac{1}{N^{k}} \sum_{m=\mu}^{k}\left(\begin{array}{c}
k-\mu \\
m-\mu
\end{array}\right)(-1)^{m} A_{N-m}^{(k)}\left(\sigma_{N-m}^{(k)}\left(z_{0}\right)-\sigma^{(k)}\left(z_{0}\right)\right) .
$$

Therefore, a sequence of polynomials in $x$ of degree not exceeding the fixed $k$ converges on the positive real axis towards $e^{-x}$. This is clearly impossible!

The proof that $S(z)$ does not belong to any $H^{p}$ space, $p>0$, will be an immediate consequence of the following proposition:

Proposition 1. If the Taylor series $S(z)=\sum_{n=0}^{\infty} a_{n} z^{n}$, convergent in $|z|<1$, defines a function in some $H^{p}$ space, $p>0$, then the series is $(C, k)$ summable at almost every point of the unit circle for an appropriate $k$ (depending only on $p$ ).

Proof of the proposition. It is enough to assume that $S(z)$ never vanishes in $|z|<1$. Indeed, considering the standard decomposition $S(z)=B(z) G(z)$, where $B(z)$ is a Blaschke product and $G(z)$ never vanishes in $|z|<1$, we can write

$$
S(z)=\frac{B(z)+1}{2} G(z)+\frac{B(z)-1}{2} G(z)=S_{1}(z)+S_{2}(z) .
$$

Thus both $S_{j}(z)$ never vanish in $|z|<1$, and it is enough to work with each $S_{j}(z)$.

First of all we observe that if $1 \leq p$, then $S(z)$ is a Fourier series and thus it is $(\mathrm{C}, \epsilon)$ summable almost everywhere on $|z|=1$, for every $\epsilon>0$ (see [9]). Also, if we accept the Carleson-Hunt Theorem, we have that if $1<p$, then $S(z)$ is $(\mathrm{C}, 0)$ summable a.e.

Next, let $\frac{1}{2}<p<1$. Write $S=S^{r} S^{t}$, where $r, t$ are chosen so that $r+t=1$ and $r<p, t<p$. Then, $S^{r} \in H^{p / r}, S^{t} \in H^{p / t}$ and they are both $(\mathrm{C}, 0)$ summable a.e.

Now we use a standard theorem (see [1], Theorem 164) saying that if two series are $(\mathrm{C}, k)$ and $(\mathrm{C}, \ell)$ summable, then their Cauchy product is $(\mathrm{C}, k+\ell+1)$ summable. Therefore $S(z)$ is $(\mathrm{C}, 1)$ summable a.e.

If $p=\frac{1}{2}$, then $r=t=\frac{1}{2}$ gives that $S(z)$ is $(\mathrm{C}, 1+\epsilon)$ summable a.e. for every $\epsilon>0$.

Proceed inductively: If $\frac{1}{k+1}<p<\frac{1}{k}$, we write $S=S^{r} S^{t}$, where $r+t=1, r<$ $k p, t<p$. Then $S^{r} \in H^{p / r}, S^{t} \in H^{p / t}$. Hence $S^{t}$ is $(\mathrm{C}, 0)$ summable a.e. and (we assume that) $S^{r}$ is $(\mathrm{C}, k-1)$ summable a.e. Therefore $S$ is $(\mathrm{C}, k)$ summable a.e. If 
$p=\frac{1}{k+1}$, the choice $r=k p, t=p$ gives that $S$ is $(\mathrm{C}, k+\epsilon)$ summable a.e. for every $\epsilon>0$.

This finishes the proof of the proposition and of Theorem 3 .

\section{REMARKS AND COMMENTS}

We initially offer two remarks on Theorem 1.

1. For simplicity in Theorem 1 we restricted ourselves to the case of integral $k$. We believe that this restriction is unnecessary and one can prove Theorem 1 for $k$ real, $k>0$, using suitably the formula of Theorem 2 ; the reader will find such kind of arguments in [5].

In [5] Marcinkiewicz and Zygmund actually proved that:

"If the series $S(z)$ is summable $(C, k+1)$ (where $k \in \mathbb{R}, k>-1$ ) at every point $z$ of a set $E \subseteq T$, to sum $\sigma^{(k+1)}(z)$, then at almost every point $z$ of $E$ the set $L^{(k)}(z)$ of limit points of the sequence $\sigma_{N}^{(k)}(z)$ is of circular structure, with center $\sigma^{(k+1)}(z)$ ".

This result suggests that Theorem 1 may be extended as follows:

Let the power series $S(z)$ converge for $|z|<1$. Also let it be $(C, k)$ summable (where $k \in \mathbb{R}, k>0$ ) for every $z$ in a certain subset $E$ of the unit circle $T$, with $(C, k)$ sum $\sigma^{(k)}(z)$. Then, for almost every $z$ of $E$, the set $L^{(m)}(z)$ of limit points of the sequence

$$
\frac{\sigma_{N}^{(m)}(z)-\sigma^{(k)}(z)}{N^{k-m-1}}, \quad N=1,2,3, \ldots, 0 \leq m<k,
$$

has circular structure with center 0 .

Observe that, although, in the case $k \neq m+1$, the actual value of $\sigma^{(k)}(z)$ plays no role in the structure of $L^{(m)}(z)$, we include it in order to cover the case $k=m+1$ (which corresponds to the theorem of Marcinkiewicz and Zygmund).

Next, we note that we can replace the factor $\left(1-\frac{z_{0}}{z_{N}}\right)^{\mu}$, appearing in the right member of the formula of Theorem 2 , by the factor $\left(\log \frac{z_{N}}{z_{0}}\right)^{\mu}$. Thus, another extension of the formula of Rogosinski is the following:

$$
\begin{gathered}
S_{N}\left(z_{N}\right)-\sigma^{(k)}\left(z_{0}\right) \\
=\left(\frac{z_{N}}{z_{0}}\right)^{N} \sum_{\mu=0}^{k}\left(\log \frac{z_{N}}{z_{0}}\right)^{\mu} \sum_{m=\mu}^{k}\left(\begin{array}{c}
k-\mu \\
m-\mu
\end{array}\right)(-1)^{m} A_{N-m}^{(k)}\left(\sigma_{N-m}^{(k)}\left(z_{0}\right)-\sigma^{(k)}\left(z_{0}\right)\right)+o(1),
\end{gathered}
$$

as $N \rightarrow \infty$.

Now we shall make some comments for the class of Universal Taylor Series. As we mentioned before, several questions arise naturally about this class of series (see [3], [4], [6] and [7]). Although some of them have been answered, there are others which remain open. For example, is a Universal Taylor series always noncontinuable across $T$ ? To establish such properties of this class of series is a natural direction of research and may be difficult, as is mentioned in [3].

On the other hand we observe that, according to the Theorem 8.37, Ch. V in [9], if $S(z)=\sum_{n=0}^{\infty} a_{n} z^{n}$ is a Universal Taylor series, then almost all the functions

$$
S_{t}(z)=\sum_{n=0}^{\infty} a_{n} z^{n} \phi_{n}(t), \quad \text { and } \quad S_{t}^{*}(z)=\sum_{n=0}^{\infty} a_{n} z^{n} \phi_{n}^{*}(t),
$$

where $\phi_{n}(t), 0<t<1$, are the sequence of Rademacher's functions and $\phi_{n}^{*}(t)=$ $\frac{1}{2}\left(1+\phi_{n}(t)\right)$, are not continuable across $T$. Moreover, for almost all $t$, the series 
$S_{t}(z)$ and $S_{t}^{*}(z)$ are not Universal, since otherwise they shall have subsequences convergent on some arc of $T$, which amounts to an application of a linear method of summation to each of them - a contradiction according to the results of paragraph 8, Chapter V in [9] (let us notice that $\sum_{n=0}^{\infty}\left|a_{n}\right|^{2}=\infty$ ). Writing now $S(z)=$ $2 S_{t}^{*}(z)-S_{t}(z)$ we see that every Universal Taylor series can be expressed as the sum of two non-Universal and not continuable Taylor series.

\section{ACKNOWLEDGEMENTS}

We would like to express our thanks to V. Nestoridis who stimulated us to work on the subject of Universal Taylor Series.

\section{REFERENCES}

[1] Hardy, G. H., Divergent Series, Oxford University Press, 1967. MR 93g:01100 (1992 reprint)

[2] Kahane, J. -P., Sur la structure circulaire des ensembles de points limites des sommes partielles d'une serie de Taylor, Acta Sci. Math. (Szeged) 45, 1-4 (1983) 247-251. MR 85f:30003

[3] Kahane, J. -P., Baire Theory in Fourier and Taylor series, Conference in the honor of Donald Newman, Philadelphia, March 1996.

[4] Kahane, J. -P., General Properties of Taylor series 1896-1996, L'Escurial, Spain, June 1996. CMP 98:09

[5] Marcinkiewicz, J. and A. Zygmund, On the behavior of trigonometric series and power series, Trans. Amer. Math. Soc. 50 (1941) 407-453. MR 3:105d

[6] Melas, A., V. Nestoridis and I. Papadoperakis, Growth of coefficients of universal Taylor series and comparison of two classes of functions, Journal d'Analyse Mathematique 73 (1997) 187202. CMP 98:10

[7] Nestoridis, V., Universal Taylor Series, Ann. Inst. Fourier, Grenoble 465 (1996) 1293-1306. MR 97k:30001

[8] Nestoridis, V. and S. K. Pichorides, The circular structure of the set of the limit points of partial sums of Taylor series, Seminaire d'Analyse Harmonique, Univ. de Paris-Sud, Mathematiques, Orsay, France (1989-90) 71-77.

[9] Zygmund, A., Trigonometric Series, second edition reprinted Vol. I, II, Cambridge University Press, 1979. MR 58:29731

Department of Mathematics, University of Crete, 71409 Heraklion - Crete, Greece

E-mail address: katsopr@talos.cc.uch.gr

E-mail address: papadim@talos.cc.uch.gr 